\title{
Lymphocyte Homing and Clinical Behavior of Non-Hodgkin's Lymphoma
}

Sirpa Jalkanen, ${ }^{*}$ Heikki Joensuu, ${ }^{\star}$ K.-O. Söderström,' and Pekka Klemi

Departments of ${ }^{*}$ Medical Microbiology, ${ }^{\ddagger}$ Radiotherapy, and ${ }^{\$}$ Pathology, University of Turku

and Turku University Central Hospital, Turku, Finland

\begin{abstract}
Lymphocyte homing receptors (HRs) defined by Hermes antibodies (anti-CD44) and lymphocyte function associated antigen-1 (LFA-1, CD11a/CD18) are involved in lymphocyte binding to endothelial cells of high endothelial venules (HEVs) at sites where lymphocytes exit the blood. Their expression was correlated to the clinical behavior of 245 non-Hodgkin's lymphomas followed up for the median of 87 mo after the diagnosis. Lymphomas that showed no or weak staining intensity for HRs were more often of stage I $(P=0.005)$, disseminated less frequently hematogenously $(P=0.003)$, and had more favorable prognosis than lymphomas with intensive staining for $H R s$ $(P<0.0001)$ despite that they were more often histologically of high grade malignancy $(P=0.002)$. Expression of LFA-1 beta chain (CD18) did not correlate significantly with stage or survival, but had prognostic value in a subgroup of $H R$ expression negative lymphomas $(P=0.03)$. HR staining intensity was an independent prognostic factor in a multivariate analysis. These findings indicate that Hermes/CD44 molecule is associated to the determination of the metastatic potential and prognosis of non-Hodgkin's lymphomas. They also reveal a new entity among non-Hodgkin's lymphomas, because lymphomas that express low levels of HR have favorable prognosis despite their often highly malignant histological appearance. (J. Clin. Invest. 1991. 87:1835-1840.) Key words: lymphocyte migration/ traffic $\bullet$ metastasis $\cdot$ CD44 $\bullet$ LFA-1
\end{abstract}

\section{Introduction}

Most mature lymphocytes circulate continuously between the blood and the lymphoid organs. Lymphocyte binding to specialized high endothelial venules (HEVs) ${ }^{1}$ is a critical step when lymphocytes leave the blood and enter the lymphatic organs (1). This step is controlled by several molecules that work in concert to establish stable contacts between the lymphocyte and the endothelium. Such molecules, based on their function, are called lymphocyte homing receptors (HRs). In human, they include the LAM-1 molecule (human equivalent for

Address correspondence and reprint requests to Dr. Sirpa Jalkanen, Dept. of Medical Microbiology, Turku University, SF-20520 Turku, Finland.

Received for publication 8 August 1990 and in revised form $21 \mathrm{No}$ vember 1990.

1. Abbreviations used in this paper: $\mathrm{HEV}$, high endothelial venule; $\mathrm{HR}$, homing receptor; LFA-1, lymphocyte function-associated antigen-1; SPF, S phase fraction; VLA-4, very late activation antigen-4.

J. Clin. Invest.

(C) The American Society for Clinical Investigation, Inc.

$0021-9738 / 91 / 05 / 1835 / 06 \$ 2.00$

Volume 87, May 1991, 1835-1840 mouse MEL-14), which mediates lymphocyte binding to peripheral lymph nodes $(2,3)$, and VLA-4, which has been described to be involved in lymphocyte traffic to mucosal HEVs (4). Lymphocyte Hermes/CD44 antigen is involved in lymphocyte binding to peripheral lymph node, mucosal, and (inflamed) synovial HEVs (5). LFA-1, a member of leukocyte integrin family, and an important adhesion molecule in a variety of immune functions, strengthens lymphocyte binding to HEVs in a non-organ-specific manner $(6,7)$.

Because the above-mentioned molecules are important in the traffic of normal lymphocytes, they might also have an essential role in dissemination of lymphoma. Recent experimental evidence supports this hypothesis. In studies of Bargatze et al. (8) the functional binding capacity of malignant lymphocytes to HEVs determined dissemination of murine lymphomas in vivo. In human studies, expression of CD44 antigen has been shown to correlate with the dissemination status of diffuse large cell lymphoma at the time of the diagnosis, but the statistical significance has varied from a trend to highly significant $(9,10)$. In our earlier study on 104 nonHodgkin's lymphomas, CD44/Hermes negative lymphomas had more favorable prognosis than Hermes positive lymphomas, and they spread less often hematogenously (11).

In the present work, we have improved the CD44/Hermes analysis by determining its expression both qualitatively (staining for CD44 absent, present, or borderline) and semiquantitatively in terms of staining intensity using tumor infiltrating $\mathrm{T}$ lymphocytes as a control and evaluated the clinical significance of CD44/Hermes HR expression in non-Hodgkin's lymphoma. In addition, we have studied the prognostic significance of CD18, which on lymphoid cells, measures the expression of LFA-1.

\section{Methods}

Patients. The hospital records of 389 adult patients diagnosed with non-Hodgkin's lymphoma in southwestern Finland and referred for treatment to the Department of Radiotherapy in Turku University Central Hospital from 1970 to 1984 were reviewed. Representative tissue was not available for review in 89 cases, in 32 cases the diagnosis was considered to be chronic lymphocytic leukemia or some other disease after a review, in 13 cases only an aspiration biopsy sample was available, and in 10 cases staging examinations were not done leaving 245 patients with histologically diagnosed non-Hodgkin's lymphoma in the series. The mean age at diagnosis was $59 \mathrm{yr}(\mathrm{SD}, 15 \mathrm{yr}$, range, $16-87 \mathrm{yr}$ ), and $133(54 \%)$ were male. 75 (31\%) patients had B symptoms, and $130(53 \%)$ had extralymphatic disease at diagnosis.

82 patients had stage I, 48 stage II, 50 stage III, and 65 stage IV disease at presentation (12). Staging included the clinical status, chest $\mathrm{x}$-ray, bone marrow biopsy, and/or aspiration biopsy. The abdomen was investigated with various combinations of ultrasound, isotope scans, bipedal lymphography, computed tomography, and laparotomy. Hematogenous spread of lymphoma was considered to be present if the dissemination pattern of lymphoma either at diagnosis, at recurrence, or at autopsy could not be explained by simple lymphatic drainage, e.g., lymphoma metastasing from a parotid gland to ipsilateral 
neck nodes, or from inquinal to iliac nodes (present, $n=154$; absent, $n$ = 87; not determined, $n=4$ ).

The patients were followed-up for the median of 87 mo after the diagnosis (range, 9-213 mo) or until death, 93\% were followed up at least for $5 \mathrm{yr}$ or until death. 95 patients were considered to have died from lymphoma during the follow-up based on clinical and/or autopsy evidence, 32 from a well-established intercurrent disease, and in 13 cases the cause of death could not be assessed with certainty. The crude 5-yr survival rate was $50 \%$, and that corrected for intercurrent deaths and deaths from an unestablished cause was $60 \%$, in stage I, $89 \%$.

Treatment was variable, but in localized cases it usually consisted of involved field or extended field radiotherapy possibly combined with combination chemotherapy, and in stages III and IV of combination chemotherapy with or without irradiation. Radiotherapy to "involved field" was given to $96(39 \%)$ patients as first-line treatment, to $34(14 \%)$ to the "mantle" field, to $25(10 \%)$ to the "inverted Y" field, and to 6 $(2 \%)$ to their combination. $84(34 \%)$ patients did not receive radiotherapy as their first-line treatment. Irradiation dose was usually about $\mathbf{4 0}$ Gy given in 4-5 wk. Combination chemotherapy with a doxorubicincontaining regimen was given to $41(17 \%)$ patients as their initial treatment, and with a nondoxorubicin-containing regimen to 104 (42\%) patients, $9(4 \%)$ patients received single-agent chlorambucil therapy, and $91(37 \%)$ patients did not receive first-line chemotherapy. Only "first-generation" regimens were used as first-line treatment, such as COP (cyclophosphamide, vincristine, prednisone), $\mathrm{CHOP}(\mathrm{H}=$ doxorubicin), MOPP (mecloretamine, vincristine, procarbazine, prednisone), and BACOP (B = bleomycin, $\mathbf{A}=$ doxorubicin).

Histology and flow cytometry. Formalin-fixed and paraffin-embedded tissue blocks were sectioned and stained with the Giemsa, hematoxylin and eosin, periodic acid-Schiff, methyl green and pyronin, and van Gieson methods. The original diagnoses were independently reviewed by two pathologists. Subclassification of the tumors was done according to the Working Formulation and Kiel classification. Hematopoietic origin of lymphomas was confirmed with a monoclonal antibody against human leukocyte common antigen (Dako, Copenhagen, Denmark). Monoclonal antibody against chicken T cells (3G6) used as serum-free culture supernatant served as a negative control antibody. The bound primary antibodies were visualized using the avidin-biotin complex technique (Vector Laboratories, Burlingame, CA) with 1,1diaminobenzidine as the chromogen. 68 lymphomas were low grade malignant according to Working Formulation (33 small cell, lymphocytic; 18 small cleaved cell, follicular; 17 mixed cell, follicular), 77 of intermediate malignancy (3 predominantly large, follicular; 27 small cleaved cell, diffuse; 30 mixed cell, diffuse; 17 large cell, diffuse), 95 of high grade malignancy (44 large cell, immunoblastic; 45 lymphoblastic; 6 small noncleaved cell) and 5 belonged to the miscellaneous category. $17(25 \%)$ of the low grade, $30(39 \%)$ of the intermediate grade, and $33(35 \%)$ of the high grade malignant lymphomas, respectively, belonged to stage I; $12(18 \%), 13(1.7 \%)$, and $22(23 \%)$ to stage II; $16(24 \%)$, $16(21 \%)$, and $18(19 \%)$ to stage III; and $23(34 \%), 18(23 \%)$, and 22 $(23 \%)$ to stage IV.

Flow cytometry was done with a FACStar ${ }^{\circledR}$ Flow Cytometer (Becton-Dickinson Immunocytometry Systems, Mountain View, CA) from deparaffinized tissue as described in detail elsewhere (11). DNA was stained with propidium iodide. S phase fraction (SPF) was calculated with the rectangular method (13).

Staining of HRs and LFA-1. CD44/Hermes expression was determined using a monoclonal mouse antibody, Hermes-3, as serum-free culture supernatant. Hermes-3 recognizes a common determinant of CD44 class of HRs mediating lymphocyte binding to peripheral lymph node, mucosal, and synovial HEVs. The production and specificity of this antibody have been described earlier (5).

Qualitative expression of the Hermes antigen was scored as - (negative), $+/-$ (a definitive population of tumor cells positive), or + (the majority or all tumor cells positive) without paying attention to the intensity of the staining.

Quantitative expression of the Hermes antigen was scored as $-1+$ (negative or very weak staining of tumor cells), ++ (intermediate inten- sity), or +++ (strong staining comparable to that of tumor infiltrating lymphocytes). Intensity was scored independently by two readers (SJ and PK). The vast majority of cases $(84.6 \%)$ clearly belonged to one of these groups, while $15.4 \%$ of samples represented so-called border line cases (either between $-/+$ and ++ or between ++ and +++ ) that were jointly reviewed and a consensus was sought. Quantification showed to be reproducible, because 35 random cases was assessed blindly twice by one reader (SJ), and the correlation coefficient between these two readings was 0.89 . A variable number of tumor infiltrating lymphocytes was seen in all cases, and they were easily recognizable with the MT1 antibody in B cell lymphomas (positive with MB2 antibody; MT1 and MB2 antibodies were from Clonab, Viereich, Germany). Because they all stained intensely with Hermes-3, they served as a useful internal staining standard for semiquantitative analysis.

A monoclonal mouse antibody (a generous gift from Prof. C. Gahmberg, University of Helsinki, 14) against the beta-subunit (CD18) of the CD11/CD18 adhesion protein complex was used to determine the expression of LFA-1 beta chain. Expression of LFA-1 beta was considered positive, if $>10 \%$ of tumor cells showed positive surface staining. LFA-1 determination was not done in 11 cases due to lack of tissue. Anti-CD18 and Hermes-3 give comparable staining patterns on fresh frozen sections and paraffin-embedded tissue sections $(11,15)$.

Coding. The patients were provided with a numerical code, and HR/LFA-1 beta analyses, histologic classification, and $S$ phase calculation were done without knowledge on survival information or other clinical data. These determinations were also done without knowledge on the results of the other analyses.

Statistical analyses. Survival analysis was done using a BMDP computer program (BMDP Statistical Software, Department of Biomathematics, University of California Press, Los Angeles, CA). Survival was estimated with the product-limit method, and comparison of survival between groups was done using the generalized Wilcoxon test (BMDP 1L). Survival rate corrected for known intercurrent deaths was used in statistical calculations. The relative importance of prognostic factors was analyzed using Cox's proportional hazard model (BMDP $2 \mathrm{~L})$. Frequency tables were analyzed using the chi-square test or Fisher's exact test. The SPF distributions of different groups were compared using Kruskal-Wallis's analysis of variance and Mann-Whitney's U-test. All $P$ values are two-tailed.

\section{Results}

24 (10\%) lymphomas were HR negative (HRexp-), 46 (19\%) borderline (HRexp+/-), and $175(71 \%)$ positive (HRexp+). When the lymphomas were scored for HR staining intensity, $77(31 \%)$ lymphomas were either negative or very weakly positive with Hermes antibody (HRint $-/+), 80(33 \%)$ showed moderate staining intensity (HRint ++ ), and $88(36 \%)$ were brightly positive (HRint +++ ). 107 (46\%) lymphomas were positive for LFA-1 beta and $127(54 \%)$ negative.

HRexp - and HRint $-/+$ lymphomas were more often of Ann Arbor stage I $(P=0.005)$ and disseminated less often hematogenously during the course of the disease $(P=0.02$ and 0.003 , respectively), although they were more often histologically high grade malignant $(P=0.02$ and 0.002 , respectively) (Table I). HRexp- and HRint-/+ lymphomas were more often of the large cell diffuse, immunoblastic, or lymphoblastic type than of any other subtype $(P=0.004$ and 0.003 , respectively). HRexp- lymphomas also had a large mean SPF ( $P$ $=0.0005$ ) as compared to HRexp+/- and HRexp+ lymphomas, but HR intensity scoring did not show significant correlation with the SPF (Table II). Neither HRexp nor HRint scoring were significantly associated with sex, presence of B symptoms or extralymphatic lymphoma at the time of the diagnosis, DNA ploidy, or DNA index, but HR negative lymphomas 
Table I. Factors that Correlate Statistically Significantly with HR Expression and Intensity in 245 Non-Hodgkin's Lymphomas

\begin{tabular}{|c|c|c|c|c|c|c|}
\hline \multirow[b]{2}{*}{ Factor } & \multicolumn{2}{|c|}{ HRexp } & \multirow[b]{2}{*}{$P$ value } & \multicolumn{2}{|c|}{ HRint } & \multirow[b]{2}{*}{$P$ value } \\
\hline & - & $+1-$ or + & & $-1+$ & ++ or +++ & \\
\hline Stage I & $11^{*}(46)^{\ddagger}$ & $71(32)$ & & $36(47)$ & $46(27)$ & \\
\hline II-IV & $13(54)$ & $150(68)$ & 0.18 & $41(53)$ & $122(73)$ & 0.005 \\
\hline \multicolumn{7}{|c|}{ Hematogenous spread } \\
\hline no & $14(58)$ & $73(34)$ & & $38(49)$ & $49(30)$ & \\
\hline yes & $10(42)$ & $144(66)$ & 0.02 & $39(51)$ & $115(70)$ & 0.003 \\
\hline \multicolumn{7}{|l|}{ Age } \\
\hline$<35 \mathrm{yr}$ & $8(33)$ & $17(8)$ & & $13(17)$ & $12(7)$ & \\
\hline$>35 \mathrm{yr}$ & $16(67)$ & $204(92)$ & 0.0009 & $64(83)$ & $156(93)$ & 0.02 \\
\hline \multicolumn{7}{|c|}{ Histological grade } \\
\hline \multicolumn{7}{|l|}{ Kiel } \\
\hline low & $7(29)$ & $118(55)$ & & $28(37)$ & $97(59)$ & \\
\hline high & $17(71)$ & $98(45)$ & 0.02 & $47(63)$ & $68(41)$ & 0.002 \\
\hline \multicolumn{7}{|l|}{ WF" } \\
\hline low & $5(21)$ & $63(29)$ & & $15(20)$ & $53(32)$ & \\
\hline interm. & $6(25)$ & $71(33)$ & & $23(31)$ & $54(33)$ & \\
\hline high & $13(54)$ & $82(38)$ & 0.30 & $37(49)$ & $58(35)$ & 0.07 \\
\hline
\end{tabular}

* Number of patients. ${ }^{\ddagger}$ Percentage of patients. ${ }^{8}$ Histological grade could be determined from 240 lymphomas. "If low and intermediate lymphomas are combined, the $P$ value is 0.12 for HR expression and 0.04 for HR intensity scoring.

were more common in patients $<35 \mathrm{yr}$ of age at the time of the diagnosis. LFA-1 beta chain expression showed no association with HRexp, HRint, or any of the factors mentioned above, but $69 \%$ of LFA-1 beta- lymphomas disseminated hematogenously during the course of the disease as compared with $58 \%$ of LFA-1 beta+ lymphomas $(P=0.06)$.

HR staining intensity correlated well with survival (Fig. 1 $A$ ). If only HRexp+ lymphomas were included in the survival analysis, HRint scoring still had considerable prognostic value (Fig. $1 B$ ). Histologically low grade, intermediate grade, and high grade malignant lymphomas could be divided into different prognostic subgroups by the HR intensity score (Fig. 2).

The survival advantage of HRexp- or exp $+/-$ lymphomas as compared with HRexp+ lymphomas $(P=0.16)$, or that of LFA- 1 beta positive lymphomas as compared with LFA-1 beta negative lymphomas $(P=0.12$, Fig. 3$)$ did not reach statistical significance. However, LFA-1 beta expression divided HRexp- lymphomas into subgroups with different outcome

Table II. Association of S Phase Fraction with Lymphocyte Homing Receptor Expression and Intensity in 230 Non-Hodgkin's Lymphomas

\begin{tabular}{cccc}
\hline$n$ & Range & Mean $(95 \% \mathrm{CI})$ & $P$ value \\
\hline$\%$ & $\%$
\end{tabular}

$\begin{array}{lrrrr}\text { HRexp } & & & & \\ - & 24 & 2.1-38.4 & 17.5(12.9-22.0) & \\ +/- & 40 & 2.3-39.7 & 13.2(10.5-15.9) & \\ + & 166 & 1.4-41.0 & 9.8(8.7-11.0) & 0.0005(- \text { vs. }+/- \text { vs. }+) \\ \text { HRint } & & & & \\ + & 69 & 2.0-39.7 & 12.9(10.6-15.2) & \\ ++ & 76 & 1.7-41.0 & 11.0(9.1-12.9) & \\ +++ & 85 & 1.4-29.6 & 10.0(8.5-11.6) & 0.24(+ \text { vs. }++ \text { vs. }+++)\end{array}$

(Fig. 4). None of the seven HRexp- LFA-1 beta positive lymphomas disseminated hematogenously during the course of the disease, whereas six of the 16 HRexp- LFA-1 beta negative lymphomas disseminated $(P=0.008)$.

To find out the relative importance and independence of the different prognostic factors, age at diagnosis $(>68 \mathrm{yr}$ vs. $\leq 68 \mathrm{yr}$, the most effective cut-off level), gender, the presence of B symptoms, presence of extralymphatic spread, WF grade (high and intermediate grade vs. low grade), Kiel grade, SPF ( $>14 \%$ vs. $\leq 14 \%$, the most effective cut-off level), stage, and HR intensity score were compared using Cox's stepwise proportional hazard model. HR staining intensity was an independent prognostic factor in a multivariate analysis $(P<0.001)$ together with the clinical stage $(P<0.001)$, Working Formulation histological grade $(P<0.001)$, gender $(P=0.004)$, size of $S$ phase fraction $(P=0.01)$, and age at diagnosis $(P=0.01$, Table III).

\section{Discussion}

$63 \%$ of the lymphomas with low staining intensity for HRs (HRint-/+) were high grade malignant according to Kiel classification, and, yet, these lymphomas had favorable prognosis (Figs. 1 and 2). Similarly, 71\% of lymphomas with no HR expression (HRexp-) were histologically high grade malignant and associated with a high S phase fraction (Table II), and despite this their prognosis was not inferior to that of HRexp+ lymphomas. These findings are at least partially explained by their lower tendency to disseminate (Table I). Because lymphomas that stain weakly for HRs (HRint-/+) have favorable prognosis despite their often highly malignant histological appearance, it is now possible to make a strong immunohistological prognostic factor by combining histological classification and HR staining intensity analysis. If this is done in the present series, the combination of Working Formulation grading and HR staining intensity becomes the strongest prognostic vari- 
A

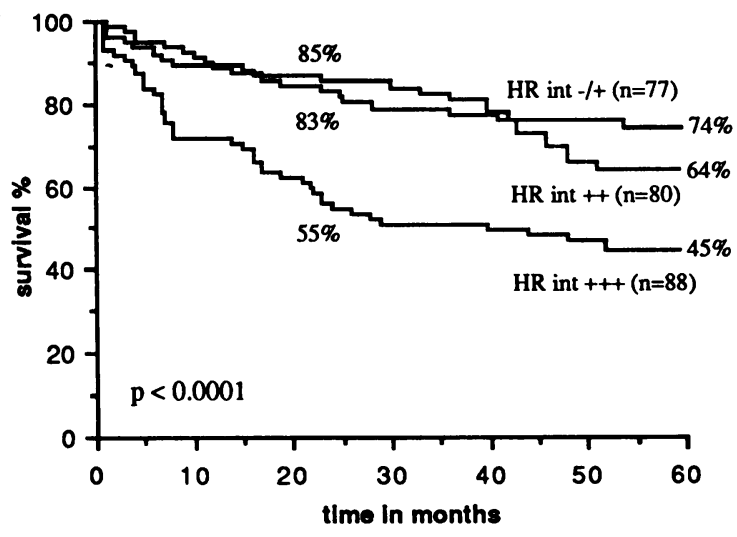

B

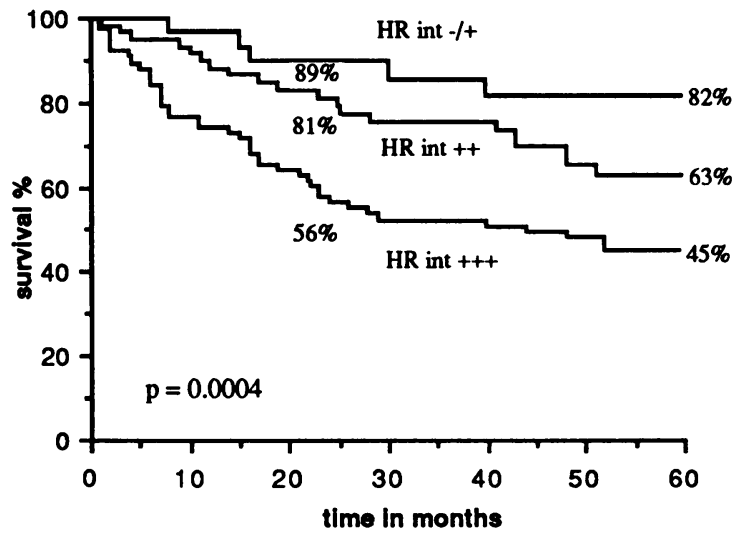

Figure 1. Survival corrected for intercurrent deaths of 245 patients with non-Hodgkin's lymphoma by staining intensity for CD44/ Hermes defined lymphocyte homing receptor (HRint score). $(A)$ Corrected survival of 175 patients with homing receptor positive lymphoma (HRexp+). Grouping is according to homing receptor staining intensity $(B)$.

able among stage II, III, and IV lymphomas in a multivariate analysis $(P=0.001$, data not shown). The decreased tendency to disseminate of lymphomas with low HR expression parallels the poor HEV binding capacity of immature lymphocytes in the bone marrow and thymus, which express low levels of CD44/Hermes antigen and do not recirculate in vivo $(1,16)$. This also indicates that lymphomas that stain weakly for CD44/Hermes functionally belong to the same group as CD44/Hermes expression negative lymphomas, even if all cells are positive. Therefore, the intensity scoring significantly improves the prognostic value of HR analysis. In our earlier work (11), based mainly on qualitative HR analysis, lymphomas in which the majority or all tumor cells were weakly CD44/ Hermes positive were grouped together with HR positive lymphomas. This does not seem to have been functionally relevant.

Lymphocyte binding to endothelial cells is likely to be a complex interaction requiring several molecules to create stable contacts between the lymphocyte and endothelial cell. LFA-1 beta expression was not significantly associated with survival (Fig. 3), and it was only marginally associated with hematogenous dissemination of lymphoma during the course of the disease $(P=0.06)$, but it could predict lymphoma dissem- ination $(P=0.008)$ and prognosis $(P=0.03)$ in the small subgroup of HRexp- lymphomas (Fig. 4). Although based on a small number of patients, this suggests that several molecules may be involved in the process of lymphoma dissemination. These probably also include LAM-1 and VLA-4, which cannot be analyzed from paraffin-embedded tissue. Involvement of these other molecules may explain the dissemination of some HRexp- lymphomas. Furthermore, HRexp- lymphomas may be heterogenous with respect to HRs, and presence of only
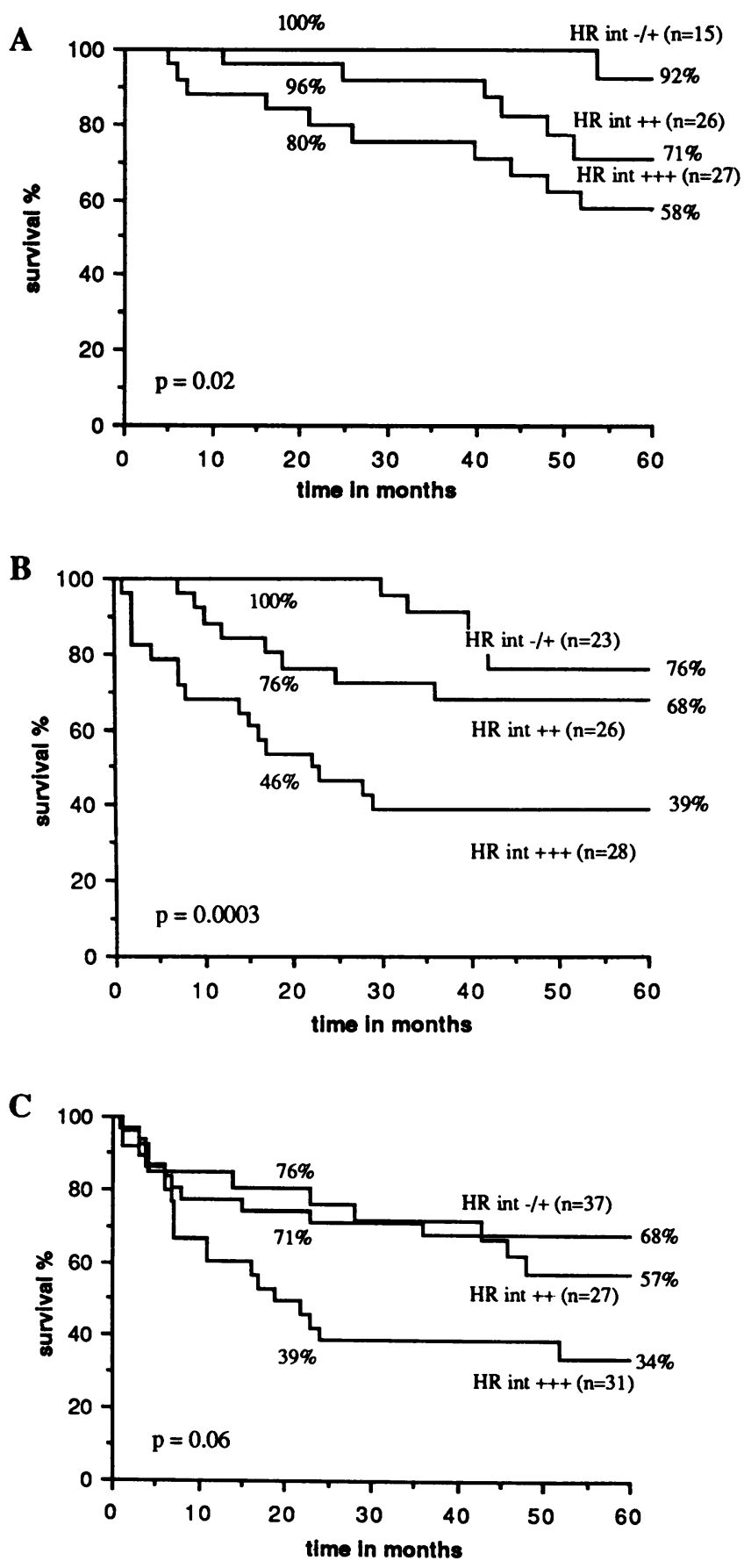

Figure 2. Effect of lymphocyte homing receptor staining intensity on survival corrected for intercurrent deaths in 68 patients with low grade $(A), 77$ with intermediate grade $(B)$, and 95 with high grade $(C)$ non-Hodgkin's lymphoma. 


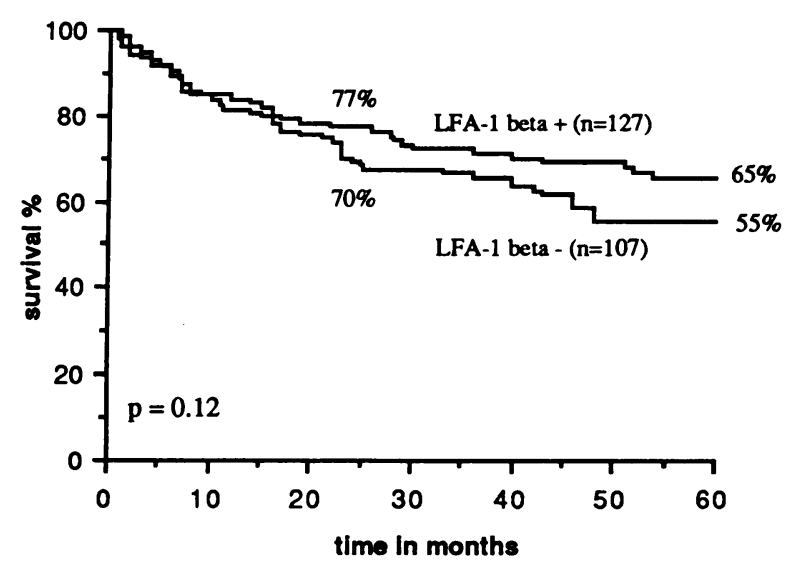

Figure 3. Survival corrected for intercurrent deaths by LFA-1 beta expression in 234 patients with non-Hodgkin's lymphoma.

a few positive lymphoma cells may result in dissemination. Also mutations may result in phenotypical transformation during the course of the disease (17).

CD44/Hermes, which is present on hematopoietic and many nonhematopoietic cell types, may have a broad role as a cell adhesion molecule (18). In addition to its involvement in lymphocyte binding to HEVs in peripheral lymph nodes, mucosa-associated lymphatic tissues, and inflamed synovium, it may mediate lymphocyte adherence to endothelium in other tissues as well. Recent findings of Oppenheimer-Marks and her colleagues (19) support this idea, because they found that CD44 is involved in mediating lymphocyte binding to IL-1 activated human umbilical vein endothelium. Moreover, lymphocyte CD44 can bind to extracellular matrix molecules such as fibronectin and hyaluronate $(20,21$, Jalkanen, S., and M. Jalkanen, submitted for publication). The ability to bind extracellular matrix molecules may be one of the determining factors in tumor invasion. Likewise, LFA-1 and VLA-4 have other functions in addition to endothelial cell binding. For example, VLA-4 also mediates lymphocyte binding to fibronectin (22, 23). Involvement of LFA-1 in cell-matrix interactions has not been reported, but it seems to be required in efficient invasion of murine lymphoma to hepatocyte and fibroblast cultures, and in vivo metastasis formation (24). Furthermore, LFA-1 mediates cell binding in many immune functions, and espe-

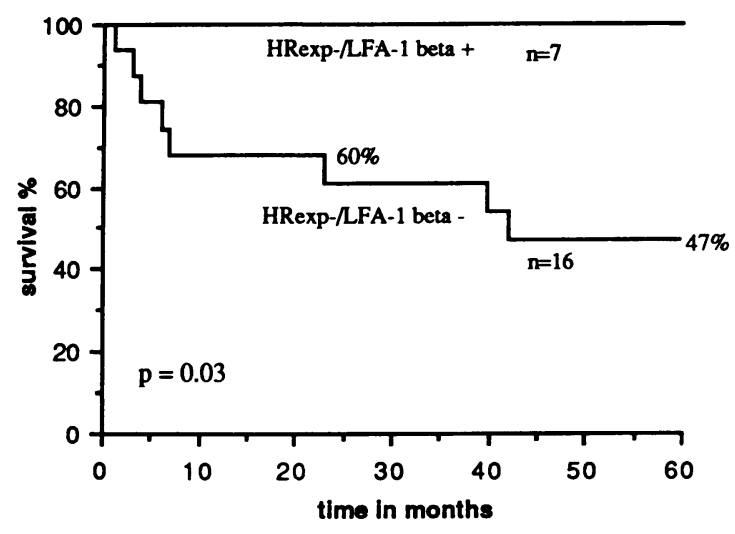

Figure 4. Survival corrected for intercurrent deaths in 23 patients with lymphocyte homing receptor expression negative (HRexp-) lymphoma by LFA-1 beta chain expression.

cially its adhesive function during $\mathrm{T}$ cell-mediated cell killing may be biologically important. The immune system has been reported to destroy more effectively high grade lymphomas with high LFA-1 expression than lymphomas that lack LFA-1 (25), which may explain why HR expression negative but LFA1 positive lymphomas were associated with good prognosis (Fig. 3). Due to these multifunctional properties of the homingassociated molecules and their complex interactions in biological progression of tumors, it is difficult to estimate all the consequences caused by the lack or overexpression of these molecules.

The possibility that more effective treatment of lymphomas that stained weakly for HR (HRint-/+) could explain their favorable outcome needs to be considered. However, there was no difference in radiotherapy given between the HRint $-/+$, HRint++, and HRint+++ lymphomas, 33 (43\%), 30 (38\%), and $33(38 \%)$ patients, respectively, were initially treated with an "involved field", $21(27 \%), 21(26 \%)$, and $23(26 \%)$ with an extended field, and $23(30 \%), 29(36 \%)$, and $32(36 \%)$ received no radiotherapy $(P=0.90)$. Similarly, there was no significant difference in chemotherapy given. 30 (39\%), $25(31 \%)$, and 36 $(41 \%)$ of patients with HRint $-/+$, HRint ++ , and HRint +++ lymphoma, respectively, did not receive chemotherapy as their first-line treatment, whereas $28(36 \%), 38(48 \%)$, and $38(43 \%)$ patients were given a nondoxorubicin containing combina-

Table III. Results of Cox's Multivariate Analysis

\begin{tabular}{|c|c|c|c|c|c|c|}
\hline & $\beta^{*}$ & $\mathrm{SE}(\beta)$ & $\beta / \mathrm{SE}$ & $\begin{array}{l}\text { Relative risk } \\
\text { of death }\left(\mathrm{e}^{\circ}\right)\end{array}$ & $P$ & $\begin{array}{l}\text { Step of factor } \\
\text { removal }\end{array}$ \\
\hline Stage & 0.61 & 0.10 & 6.13 & $1.84(1.51-2.25)$ & $<0.001$ & 1 \\
\hline Histological grade & 0.55 & 0.15 & 3.73 & $1.73(1.30-2.32)$ & $<0.001$ & 2 \\
\hline HRint score & 0.60 & 0.15 & 4.09 & $1.82(1.36-2.44)$ & $<0.001$ & 3 \\
\hline Sex & 0.81 & 0.23 & 3.46 & $2.24(1.43-3.35)$ & 0.004 & 4 \\
\hline$S$ phase fraction & 0.72 & 0.25 & 2.93 & $2.05(1.26-3.35)$ & 0.01 & 5 \\
\hline Age at diagnosis & 0.02 & 0.01 & 2.46 & $1.02(1.00-1.04)$ & 0.01 & 6 \\
\hline Kiel grade & - & - & - & - & $\mathbf{N S}^{\ddagger}$ & - \\
\hline Extralymphatic spread & - & - & - & - & - & NS \\
\hline B symptoms & - & - & - & - & NS & - \\
\hline
\end{tabular}

* The $\beta$ coefficient describes how each factor contributes to the hazard, and $\beta / \mathrm{SE}$ (standard error) describes their significance ( $z$ value). The relative risk of death $\left(\mathrm{e}^{\beta}\right)$ is given with $95 \%$ confidence intervals. ${ }^{\ddagger} \mathrm{NS}$, not significant. 
tion, and $17(22 \%), 13(16 \%)$, and $11(13 \%)$ patients, respectively, received a doxorubicin containing regimen $(P=0.34)$. In addition, 2, 4, and 3 patients, respectively, were first treated with single-agent chlorambucil.

In conclusion, the results indicate that molecules involved in normal lymphocyte recirculation may also be important in migration of their malignant counterparts, and suggest that lymphocyte HR determination is of value in the histological evaluation of non-Hodgkin's lymphomas. CD44/Hermes antigen analysis reveals a new entity among non-Hodgkin's lymphomas. HR expression negative lymphomas (HRexp-), and lymphomas that do not stain intensely for HR (HRint-/+) form a subgroup characterized by high histological grade and large S phase fraction (HRexp- lymphomas), but decreased tendency for dissemination and generally favorable prognosis. Because high grade malignant lymphomas are often treated with aggressive chemotherapy with toxicity that may be lifethreatening, identification of a subgroup with less serious prognosis and tendency to remain local may be of therapeutic importance. Analysis of other lymphocyte homing-associated molecules may further improve the assessment of prognosis and migration of lymphoma cells to different anatomical sites.

\section{Acknowledgments}

The authors thank Prof. Carl Gahmberg for anti-LFA-1 beta antibody, Dr. Juhani Tuominen for statistical advice, and Maritta Pohjansalo for technical help.

This work was supported by grants from the Finnish Cancer Foundation, the Sigrid Juselius Foundation, the Finnish Academy, and the Turku University Foundation.

\section{References}

1. Butcher, E. C. 1986. The regulation of lymphocyte traffic. Curr. Top. Microbiol. Immunol. 128:85-122.

2. Gallatin, W. M., E. C. Butcher, and I. L. Weissman. 1983. A cell surface molecule involved in organ-specific homing of lymphocytes. Nature (Lond.). 304:30-34.

3. Tedder, T. F., C. M. Isaacs, T. J. Ernst, G. D. Demetri, D. A. Afler, and C. M. Disteche. 1989. Isolation and chromosomal localization of cDNAs encoding a novel lymphocyte cell surface molecule, LAM-1. J. Exp. Med. 170:123-133.

4. Holzmann, B., and I. L. Weissman. 1989. Integrin molecules involved in lymphocyte homing to Peyer's patches. Immunol. Rev. 108:45-62.

5. Jalkanen, S. R. Bargatze, J. de los Toyos, and E. C. Butcher. 1987. Lymphocyte recognition of high endothelium: antibodies to distinct epitopes of an 85-95 kD glycoprotein antigen differentially inhibit lymphocyte binding to lymph node, mucosal, or synovial endothelial cells. J. Cell Biol. 105:983-990.

6. Hamann, A., D. Jablonski-Westrich, A. Duijvestijn, E. C. Butcher, H. Baisch, R. Harder, and H. G. Thiele. 1988. Evidence for an accessory role of LFA-1 in lymphocyte-high endothelium interaction during homing. J. Immunol. 140:693-699.
7. Pals, S. T., A. den Otter, F. Miedema, P. Kabel, C. D. Keizer, R. J. Scheper, and C. J. L. M. Meijer. 1988. Evidence that leukocyte function-associated antigen-1 is involved in recirculation and homing of human lymphocytes via high endothelial venules. J. Immunol. 140:1851-1853.

8. Bargatze, R. F., N. Wu, I. L. Weissman, and E. C. Butcher. 1987. High endothelial venule binding as a prediction of the dissemination of passaged $\mathrm{mu}$ rine lymphomas. J. Exp. Med. 166:1125-1131.

9. Picker, L. J., L. J. Medeiros, L. M. Weiss, R. A. Warnke, and E. C. Butcher. 1988. Expression of lymphocyte homing receptor antigen in non-Hodgkin's lymphoma. Am. J. Pathol. 130:496-504.

10. Pals, S. T., E. Horst, G. Ossekoppele, C. G. Fidgor, R. J. Scheper, and C. J. L. M. Meijer. 1989. Expression of lymphocyte homing receptor as a mechanism of dissemination in non-Hodgkin's lymphoma. Blood. 73:885-888.

11. Jalkanen, S., H. Joensuu, and P. Klemi. 1990. Prognostic value of lymphocyte homing receptor and $S$ phase fraction in non-Hodgkin's lymphoma. Blood. 75:1549-1556.

12. Carbone, P. P., H. S. Kaplan, K. Musshoff, D. W. Smithers, and M. Tubiana. 1971. Report of the committee on Hodgkin's disease staging classification. Cancer Res. 31:1860-1861.

13. Camplejohn, R. S., J. C. Macartney, and R. W. Morris. 1989. Measurement of $S$ phase fractions in lymphoid tissue comparing fresh versus paraffin-embedded tissue and 4,6-diamino-2 phenylindole dihydrochloride versus propidium iodide staining. Cytometry. 10:410-416.

14. Nortamo, P., M. Patarroyo, C. Kantor, J. Suopanki, and C. Gahmberg. 1988. Immunological mapping of the human leukocyte adhesion glycoprotein GP90 (CD18) by monoclonal antibodies. Scand. J. Immunol. 28:537-546.

15. Jalkanen, S., R. Aho, M. Kallajoki, T. Ekfors, A. Duijvestijn, and H. Kalimo. 1989. Lymphocyte homing receptors and adhesion molecules in intravascular malignant lymphomatosis. J. Int. Cancer. 44:777-782.

16. Jalkanen, S., R. Bargatze, L. Herron, and E. C. Butcher. 1986. A lymphoid cell surface glycoprotein involved in endothelial cell recognition and lymphocyte homing in man. Eur. J. Immunol. 16:1195-1202.

17. Joensuu, H., P. Klemi, and S. Jalkanen. 1990. Biological progression in non-Hodgkin's lymphoma. A flow cytometric study. Cancer (Phila.). 65:25642571

18. Picker, L. J., M. Nakache, and E. C. Butcher. 1989. Monoclonal antibodies to human lymphocyte homing receptors define a novel class of adhesion molecules on diverse cell types. J. Cell Biol. 109:927-938.

19. Oppenheimer-Marks, N., L. S. Davis, and P. E. Lipsky. 1990. Human T lymphocyte adhesion to endothelial cells and transendothelial migration. Alteration of receptor use relates to the activation status of both the $T$ cell and the endothelial cell. J. Immunol. 145:140-148.

20. Miyake, K., C. B. Underhill, J. Lesley, and P. Kincade. 1990. Hyaluronate can function as a cell adhesion molecule and CD44 participates in hyaluronate recognition. J. Exp. Med. 172:69-76.

21. Aruffo, A., I. Stamenkovic, M. Melnick, C. B. Underhill, and B. Seed 1990. CD44 is the principal cell surface receptor for hyaluronate. Cell. 61:13031313.

22. Wayner, E. A., A. Garcia-Pardo, M. J. Humpries, J. A. McDonald, and W. G. Carter. 1989. Identification and characterization of the T lymphocyte adhesion receptor for an alternative cell attachment domain (CS-1) in plasma fibronectin. J. Cell Biol. 109:1321-1330.

23. Guan, J.-L., and R. O. Hynes. 1990. Lymphoid cells recognize an alternatively spliced segment of fibronectin via the integrin receptor alfa4/betal. Cell. 60:53-61.

24. Roossien, F. F., D. Rijk, A. Bikker, and E. Roos. 1989. Involvement of LFA-1 in lymphoma invasion and metastasis demonstrated with LFA-1-deficient mutants. J. Cell Biol. 108:1979-1985.

25. Clayberger, C., L. J. Medeiros, M. P. Link, R. A. Warnke, A. Wright, T. D. Koller, S. D. Smith, and A. M. Krensky. 1987. Abscence of cell surface LFA-1 as a mechanism of escape from immunosurveillance. Lancet. ii:533-536. 